\title{
Reconstrucción cognitiva de los conceptos centrales de la función exponencial: un estudio de enfoque mixto
}

\author{
Miguel A. Rodríguez ${ }^{1,2 *}$, Carlos A. Ledezma ${ }^{3}$, Andrea S. Vergara ${ }^{2,4}$, Pablo Gregori $^{5}$ \\ (1) Facultad de Educación, Univ. Playa Ancha, Playa Ancha 850, Valparaíso-Chile \\ (2) Instituto de Matemática, Pontificia Univ. Católica de Valparaíso, Blanco Viel 596, Valparaíso-Chile \\ (correo-e: mrodriguez@upla.cl; correo-e: miguel.rodriguez@pucv.cl) \\ (3) Departamento de Educación, Univ. Barcelona, Passeig de la Vall d'Hebrón, 171, Barcelona-España \\ (correo-e: cledezar25@alumnes.ub.edu) \\ (4) Facultad de Educación, Univ. Academia de Humanismo Cristiano, Catedral 1721, Santiago-Chile \\ (correo-e: andrea.vergara@uacademia.cl; andrea.vergara@pucv.cl) \\ (5) Instituto Universitario de Matemática de Castellón, Departamento de Matemática Univ. Jaume I, Av. de Vicente Sos Baynat, \\ s/n, Castellón de la Plana-España (correo-e: gregori@uji.es)
}

Recibido May. 6, 2021; Aceptado Jul. 5, 2021; Versión final Ago. 16, 2021, Publicado Dic. 2021

\section{Resumen}

Este estudio presenta evidencias sobre la validación de un modelo cognitivo llamado descomposición genética para la reconstrucción del concepto función exponencial. Se encuesta a 22 estudiantes del profesorado de matemática y de maestría en didáctica de la matemática mediante la aplicación de un cuestionario desde un enfoque de metodología mixta. Se muestra evidencia de cómo los participantes del estudio utilizan de forma paralela el operador logaritmo y la función exponencial. Entre los resultados se destaca el rol que desempeña la gráfica, el uso de patrones y la potenciación para establecer las restricciones a la base de la relación exponencial. Sumado a lo anterior, se confirma el rol que desempeña la multiplicación recursiva de un número real positivo para poner de relieve la monotonía de la función exponencial. Se concluye que estos resultados permiten validar el modelo cognitivo propuesto de descomposición genética, vindicando los elementos teóricos que lo sustentan.

Palabras clave: descomposición genética; estadística implicativa; función exponencial; formación docente; teoría APOE

\section{Cognitive reconstruction of the core concepts of the exponential function: a mixed approach study}

\begin{abstract}
This study presents evidence that validates a cognitive model called genetic decomposition for the reconstruction of the exponential function concept. Twenty-two students enrolled in a mathematics teaching degree and in a master's degree in didactics of mathematics are surveyed through the application of a questionnaire using a mixed methodology approach. The results show that the students surveyed use both the logarithm operator and the exponential function in parallel. The results underscore the roles played by graphs and the use of patterns and the power operation to establish restrictions at the base of the exponential relationship. In addition, the results confirm the role played by recursive multiplication of a positive real number to highlight the monotony of the exponential function. It is concluded that these results validate the proposed model of genetic decomposition, vindicating the theoretical elements that support it.
\end{abstract}

Keywords: genetic decomposition; implicative statistics; exponential function; teacher training; APOE theory 


\section{INTRODUCCIÓN}

Si bien la función exponencial posee una mayor tradición que la función logaritmo en el ámbito escolar, históricamente los logaritmos son descubiertos antes que los exponentes (Eves, 1976). En efecto, cuando Euler introduce el uso de la función exponencial, lo hace apoyándose de los logaritmos, los cuales resuelven el problema de la uniformización o estandarización de la expresión exponencial (Cajori, 1913). De acuerdo con indagaciones didácticas de estos tópicos, exponenciales y logaritmos se desarrollaron para simplificar y promover cálculos en el modelamiento de diversos fenómenos (Ferrari y Farfán, 2010). En relación a eso último, la función exponencial puede ser abordada desde una perspectiva constructivista, teniendo en cuenta que, cuando el crecimiento de la variable independiente corresponde a una progresión aritmética, la variable dependiente expresa su crecimiento mediante una progresión geométrica (Confrey, 1991).

Por otra parte, desde el punto de vista tanto cognitivo como epistemológico, existen distintas investigaciones que han abordado de forma específica algunas nociones gravitantes para el aprendizaje de la función exponencial. Por ejemplo, se han analizado las dificultades que surgen en los estudiantes en relación con el uso de las convenciones matemáticas presentes en el tratamiento de los exponentes, según el sistema numérico de referencia (Martínez-Sierra, 2002); de similar manera se ha estudiado cómo la modelación de ciertos fenómenos con apoyo de graficadores puede contribuir a la comprensión del comportamiento exponencial (Confrey y Smith, 1994). A la luz de lo que se ha descrito, nos proponemos abordar, desde un punto de vista cognitivo, la reconstrucción de los conceptos centrales de la función exponencial —multiplicación recursiva de un número real, potenciación, invariante exponencial, monotonía, entre otras- en estudiantes de pedagogía en matemática y de maestría en Didáctica de la Matemática. La idea de reconstrucción se justifica en la medida que los informantes han aprendido previamente estos conceptos, tanto en sus procesos de formación de enseñanza secundaria como terciaria, tomando en cuenta que algunos de ellos enseñan dicho concepto a nivel escolar. El concepto función exponencial permite articular la matemática escolar y la matemática universitaria mediante nociones como variable, potencia, raíz, progresión geométrica y logaritmo. Además sirve de modelo para aplicaciones en otras áreas del conocimiento en ambos niveles educacionales. Por lo tanto nuestra motivación es avanzar en este primer tránsito, ya que el paso de exponentes racionales a reales es una dimensión que se aleja de la educación escolar y no es trivial, un aspecto que deseamos abordar más adelante en función de los resultados de este estudio.

Es precisamente debido a esas articulaciones que avizoramos pertinente indagar en los mecanismos y estructuras mentales que son fundamentales para el aprendizaje de dicho concepto matemático, ya que nuestros informantes tendrán, o tienen a cargo, enseñar dicho concepto a nivel escolar. Por último, cabe indicar que para esta investigación asumimos como relación exponencial entre las variables $x$ e $y$ a la igualdad $y=a^{x}$, donde su base a y el exponente $x$ asumen cualquier valor real. Por otro lado, la función exponencial representa un caso particular de la relación exponencial, la que cumple con propiedades específicas como la continuidad e inversión con el logaritmo, y restricciones en cuanto al dominio y el valor que asume su base.

Algunas investigaciones en Matemática Educativa han abordado particularmente el aprendizaje de la función exponencial a través del modelado de fenómenos reales de crecimiento y decrecimiento, focalizando su estudio en la forma que los estudiantes comprenden y articulan el comportamiento de las variables involucradas (Bryan, 2012; Bush et al., 2015; Ellis et al., 21015; Guerrero-Ortiz et al., 2018) —por ejemplo Bryan (2015) reporta una actividad para escolares que involucra la recopilación de datos sobre el decrecimiento de una sustancia radiactiva, donde pone de relieve el uso de funciones exponenciales mediante los registros tabular y gráfico. En Bush et al. (2015) proponen una actividad para introducir funciones exponenciales mediante diferentes registros, registro tabular, registro algebraico y el registro gráfico, para analizar la conveniencia de aquellos registros en el estudio de la propagación de una epidemia. Por otro lado en Ellis et al. (2015) reportan un experimento de enseñanza donde los escolares analizan el crecimiento de una planta mediante el uso del registro tabular. Por último, en Guerrero-Ortiz et al. (2018) reportan un estudio a nivel de formación de profesores de matemáticas con tareas que demanda un ciclo de modelación en el crecimiento y decrecimiento de variables que se relacionan con la función exponencial. Otros estudios se han centrado en el razonamiento que se hace de la función exponencial desde un punto de vista covariacional, enfatizando el rol dinámico que ejerce la noción tasa de cambio en la caracterización de la variación (Confrey y Smith, 1994; Ferrari-Escolá et al., 2016). Las relaciones exponenciales también han sido estudiadas teniendo en cuenta el ámbito específico de la matemática en el que son desplegadas las tareas que atienden sus aprendices. Por ejemplo, Presmeg y Nenduradu (2005) se centran en la resolución de problemas algebraicos que involucran exponenciales, examinando críticamente los usos de distintos registros de representación. Cabe mencionar que en dicho estudio se reporta el uso de la covariación mediante un modelo lineal, poniendo de manifiesto la persistencia del pensamiento proporcional por parte de un estudiante. 
En Montoya-Delgadillo et al. (2018), se enfocan en la función exponencial como un objeto matemático propio del análisis, discutiendo su aprendizaje en tareas asociadas a límites de funciones. Desde otro punto de vista, se ha explorado el surgimiento histórico de algunos métodos asociados a exponenciales, con el propósito de reconsiderar las formas no convencionales con las que los estudiantes abordan esta noción (Dennis y Confrey, 1996). Todos los aspectos que se han explicitado orientan y justifican la selección de tareas que dan forma a nuestro cuestionario que presentamos más adelante en el apartado metodológico. En distinción a las perspectivas descritas, en la Tabla 2 se asume una mirada conjunta de las posibles estrategias, procedimientos y argumentos a priori que permiten la reconstrucción de la función exponencial como objeto matemático y, a su vez, diseñamos un modelo cognitivo que incluye distintos estados de construcción para los conceptos previos, asociados a la función exponencial los que se interpretarán en atención a los insumos del enfoque estadístico que se describe en el apartado metodológico desde el modelo cognitivo que proponemos.

En atención a lo que ya hemos expuesto, nuestro objetivo es diseñar y validar un modelo cognitivo para la reconstrucción de la función exponencial. Por otro lado, nuestras preguntas de investigación son las siguientes: (i) ¿Qué procedimientos matemáticos, estrategias y argumentos contribuyen a la reconstrucción del concepto función exponencial en estudiantes de pedagogía en matemática y maestría?; y (ii) ¿cómo dichos elementos se relacionan con el modelo cognitivo para el aprendizaje de este concepto en los procesos de formación del profesorado de matemática?

\section{OTROS ANTECEDENTES}

La Teoría APOE (acrónimo de Acción, Proceso, Objeto y Esquema) fue concebida para explicar cómo se aprenden conceptos matemáticos, para lo cual se describen las construcciones y mecanismos mentales involucrados en el aprendizaje de tales conceptos (Arnon et al., 2014). Las cuatro construcciones mentales que caracterizan a esta teoría son: acción, proceso, objeto y esquema. Cabe indicar que dichas construcciones se relacionan estrechamente con mecanismos de abstracción reflexiva, tales como interiorización, coordinación, encapsulación, desencapsulación, generalización y reversión (Arnon et al., 2014). Dichas estructuras mentales son la base para construir un esquema de un concepto, concebido este como una colección coherente de acciones, procesos, objetos y esquemas de otros conceptos. El nivel de desarrollo de un esquema se establece según el tipo de relaciones que se puede dar entre los componentes que lo constituyen, lo que se evidencia en cómo un individuo aborda un determinado problema matemático. Para ello, se han establecido tres niveles de coherencia para un esquema -intra, inter, trans-, el cual puede ser tematizado en un nuevo objeto. En la Figura 1 se presenta un panorama general que relaciona las estructuras y mecanismos mentales involucrados en la construcción de un concepto o de una porción de conocimiento matemático.

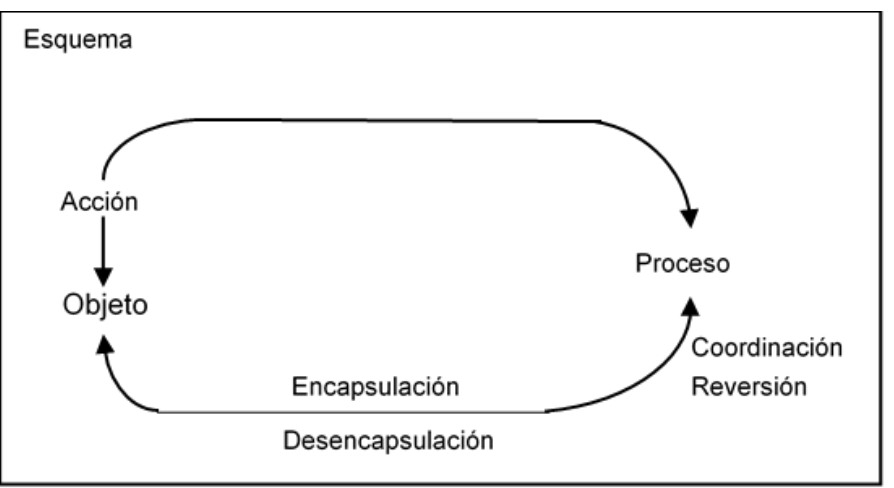

Fig. 1: Estructuras y mecanismos mentales para la construcción de un concepto (adaptada de Arnon et al., 2014, p. 18)

Una acción es una estructura mental de naturaleza algorítmica que obedece a estímulos externos (Arnon et al., 2014). Un aprendiz ha interiorizado una acción en un proceso cuando puede realizar dicha operación en su mente, sin tener que realizar todos los pasos específicos. Una acción es una operación mental que no obedece a estímulos externos, es una construcción dinámica. Un proceso puede revertirse en otro proceso, y dos procesos pueden coordinarse para construir un nuevo proceso. Si un aprendiz considera un proceso como un todo, realizando acciones sobre él, se considera que ha encapsulado el proceso en un objeto. Un elemento importante en la teoría APOE, y que actúa como hipótesis de investigación, es la Descomposición Genética (DG), modelo que describe en detalle las construcciones y mecanismos mentales que son necesarios para que un estudiante aprenda de manera significativa un concepto matemático. 
Dicho modelo no pretende ser único y debe ponerse a prueba experimentalmente para ser validado o refinado, a través de la aplicación de técnicas de recolección de datos y el posterior análisis (Arnon et al., 2014). Los principales elementos que fundamenten una descomposición genética son la experiencia del investigador (Dubinsky y Harel, 1992), la epistemología del concepto (Cordero y Miranda, 2002) y la identificación de los procesos utilizados por los estudiantes, a través de investigaciones previas (Dubinsky y Lewin, 1986). En esta investigación la DG se ha diseñado considerando esos tres elementos. La Teoría APOE también proporciona un ciclo de investigación compuesto por tres fases: análisis teórico (diseñar una DG), diseño y aplicación de instrumentos, y el análisis y verificación de los datos. Este ciclo ha sido utilizado con éxito por el grupo RUMEC (Research in Undergraduate Mathematics Education Community) y otros investigadores (González y RoaFuentes, 2017; Rodríguez et al., 2019).

\section{La teoría APOE y la función exponencial}

De las investigaciones referidas a la función exponencial que han utilizado la teoría APOE destacamos el trabajo de Weber (2002), quien propone un conjunto de construcciones mentales para que un estudiante pueda comprender en profundidad la exponenciación así como la aplicación del operador logaritmo en el ámbito de una ecuación exponencial. En particular propone iniciar con la función exponencial y logarítmica con dominio en $\mathbf{N}$. Además plantea que la construcción de la función exponencial se logra a través de interiorizar la acción multiplicación recursiva de un real positivo en el proceso exponenciación, el que a su vez debe revertirse en el proceso logaritmo mediante la resolución de una ecuación exponencial. El autor propone además que tanto la función exponencial como la logarítmica deben construirse como procesos a partir de la generalización del proceso exponenciación, ampliando el sistema de los números naturales a los racionales, siendo este el foco de su propuesta para permitir a los estudiantes un aprendizaje no memorístico de la función exponencial. Dicho estudio concluye que los 15 estudiantes universitarios, quienes participaron de un ciclo de enseñanza en un curso clásico de pre-cálculo, sólo alcanzan una concepción acción de función exponencial, imposibilitándolos poder explicar el porqué de determinadas reglas de transformación asociadas a los exponentes. En definitiva, se evidencia la necesidad de coordinar procesos asociados a la exponenciación, el operador logaritmo y la función exponencial.

Por otro lado, Vargas (2012) plantea que para construir el concepto función exponencial como esquema, se hace necesario la construcción de la invariante exponencial que relaciona la covariación de las variables asociadas a dicha función, considerando además los esquemas de límite, continuidad y el número e (Vargas, 2012). En particular se enfoca en describir los mecanismos de interiorización, encapsulación y tematización mediante la enseñanza. Con ello distingue entre los elementos globales, los que asocia con las distintas formas de concebir la función exponencial y sus diferentes parámetros desde un punto de vista analítico. Además de los elementos puntuales, asociados principalmente con aspectos epistemológicos, como la distinción de la existencia de dos funciones exponenciales que se distinguen una de otra por el tipo de monotonía. Con ello pone de relieve la conexión que se debe dar entre la función exponencial y logarítmica. Se agrega a dicha propuesta la tematización del esquema considerando el estudio de fenómenos de crecimiento y decrecimiento.

\section{Descomposición genética de la función exponencial}

Nuestra propuesta de DG, para construir la función exponencial como objeto, considera, entre otros, la multiplicación recursiva como acción y el proceso exponenciación que plantea Weber (2002). Respecto de la propuesta de Vargas (2012) consideramos la invariante exponencial. Así nuestra DG procede de la siguiente manera: se debe repetir la acción asignar una posición a la multiplicación recursiva (con exponente natural) de un número real positivo. Ello demanda que cada nuevo producto se multiplique por el número inicial para que, mediante la multiplicación de potencias de igual base, se interiorice dicha acción en el proceso progresión exponente. Por otro lado, es necesario desencapsular el objeto variable real en el proceso variable dependiente, al establecer una relación entre la posición de cada nuevo producto con el respectivo exponente que se va generando. Los procesos progresión exponente y variable dependiente se deben coordinar en el proceso relación exponente natural, generalizando, mediante una ecuación, los listados que representan a una variable dependiente e independiente. Por otro lado, es necesario desencapsular el objeto potenciación en el proceso exponente racional, al relacionar un exponente fraccionario con el operador raíz. Así, los procesos relación exponente y exponente racional se coordinan en el proceso invariante exponencial, que se refiere a las diferencias entre los valores de la variable independiente y los respectivos cocientes para la variable dependiente, mediante el uso de exponentes. El proceso invariante exponencial se coordina con el proceso operador logaritmo, resultando el proceso función exponente. Luego, se requiere que el objeto función se debe desencapsular en el proceso función inversa, cuando se analizan los casos en que la relación exponente define una función. Los 
procesos función inversa y función exponente se deben coordinar en el proceso función exponente. Por último, el proceso función exponente se encapsula en el objeto función exponencial, cuando se generaliza la invariante exponencial en el sistema de los números reales. En el diagrama de la Figura 2 damos a conocer como se relacionan las construcciones y mecanismos mentales que hemos propuesto.

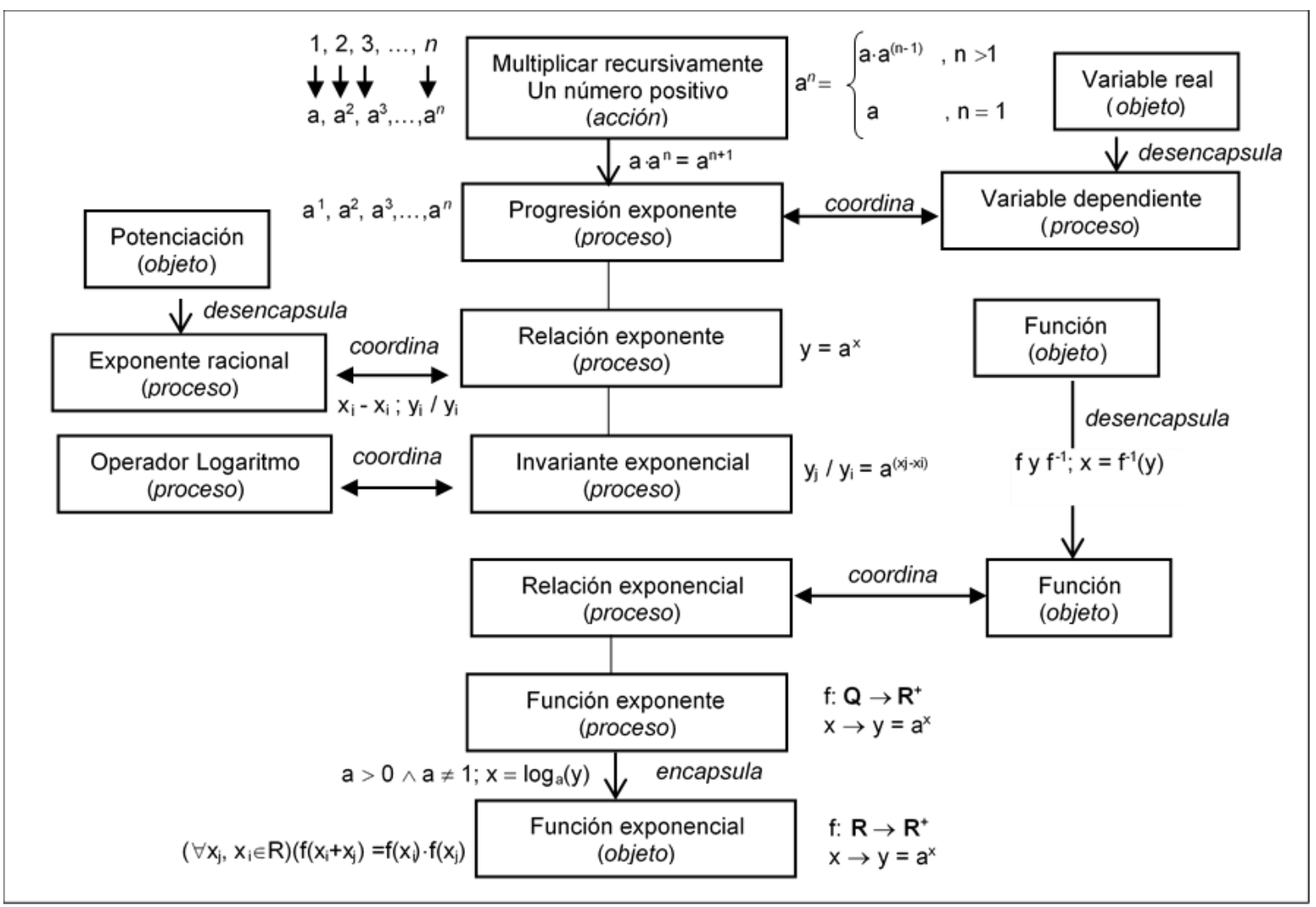

Fig. 2: Diagrama de la descomposición genética

Es importante destacar que nuestro estudio se enfoca en modelar el tránsito del álgebra escolar al álgebra universitaria, por lo tanto nuestra descomposición genética se centra en la influencia de conocimiento escolar a diferencia de lo que plantean Vargas y Weber, quienes asumen la reconstrucción de la función exponencial con base en un conocimiento más acabado de los conocimientos previos

\section{METODOLOGÍA}

La investigación es de carácter mixto, ya que combina un estudio de caso instrumental (Stake, 2010) y el uso de la estadística implicativa (Gras et al., 2008), bajo el marco general de análisis que provee el ciclo metodológico de la teoría APOE. Se consideraron dos casos de estudio, estudiantes de Pedagogía en Matemáticas y estudiantes de Maestría en Didáctica de la Matemática. Cabe indicar que los informantes del curso de maestría se desempeñaban como profesores de matemáticas en el sistema escolar y uno de los investigadores, que realiza docencia en dicho programa, pudo acceder a ellos para aplicar el cuestionario. Los estudiantes de pedagogía cursaban sus estudios en una universidad pública de una región en Chile y fueron contactados por una exestudiante del programa que es docente en dicha universidad. Con dicho enfoque nos propusimos indagar en profundidad el nivel de comprensión de dicho concepto en dos realidades específicas, a saber, en la formación inicial y continua. En ambos casos los informantes habían experimentado el aprendizaje de la función exponencial a nivel escolar y en un curso de álgebra a nivel universitario. La estadística implicativa la utilizamos para identificar asociaciones significativas entre las categorías de análisis a priori con el propósito de reportar datos claves e informantes de interés.

Los resultados del análisis estadístico en general los usamos para orientar de manera más objetiva los énfasis que requiere la interpretación cualitativa de los datos, la que se realiza siguiendo la teoría APOE. La aplicación del cuestionario no implicó instancias de inducción en el tema en cuestión y no hubo indicaciones por parte de quienes aplicaron el instrumento. Con la primera actividad se esperaba que los estudiantes pudiesen identificar 
la invariante exponencial y la pudiesen aplicar en la preguntas 1 y 3 . Con la pregunta 1 se pretendió activar aspectos relacionados con la exponenciación como plantea Weber (2002) y con la pregunta 2 aspectos más específicos de la función exponencial desde el sistema de los números racionales, en el sentido de lo que plantea Vargas (2012).

Es precio indicar que desde el enfoque metodológico que hemos asumido no es necesario realizar un muestreo estadístico con un gran número de informantes, pues no se pretende una generalización de los resultados obtenidos, sino más bien mostrar evidencias de una construcción específica (Rodríguez et al., 1999). Por último, para validar la DG, de la Figura 2, se diseñó y aplicó un cuestionario de preguntas abiertas que fueron validadas a través del juicio de tres expertos, especialistas en la teoría APOE y con grado de doctor en Didáctica de la Matemática. En esta validación externa, los expertos se pronunciaron acerca de la claridad, pertinencia y coherencia de las preguntas del cuestionario respecto de la DG.

\section{Sobre el estudio de caso y el cuestionario}

Nuestros dos casos de estudio incluyen a 22 estudiantes los que fueron rotulados con una letra y un número. Para el caso 1 que incluye a 13 estudiantes del profesorado de matemática que designaremos de P1 a P13 y caso 2, son 9 estudiantes de maestría en Didáctica de la Matemática que designaremos de M1 a M9. A continuación, en la Tabla 1, presentamos el cuestionario que incluye una actividad y tres problemas.

Tabla 1. Cuestionario para validar la descomposición genética.

\section{Actividad:}

Multiplique recursivamente un número real positivo distinto de 1 y a continuación:

a) Construya una tabla de valores con los datos obtenidos y escriba una ecuación que relacione ambas variables. Explique sus procedimientos.

b) De la tabla anterior, escoja distintos pares de valores (contiguos y no-contiguos) para la variable independiente y calcule la diferencia entre éstos (en ambos sentidos). Para el respectivo par de la variable dependiente, calcule el cociente de dichos valores, expresándolos en forma fraccionaria. Finalmente, enuncie una conjetura -en lenguaje matemático- que relacione la diferencia y el cociente.

\section{Problema 1}

Considere la siguiente tabla de valores:

\begin{tabular}{|c|c|c|c|c|c|}
\hline$x$ & -2 & -1 & 0 & 4 & 8 \\
\hline$y$ & $1 / 3$ & $3^{1 / 2} / 2$ & 1 & 9 & 81 \\
\hline
\end{tabular}

a) Escriba una ecuación que relacione las variables de la tabla. Fundamente sus procedimientos.

b) Obtenga una expresión de $x$ en función de $y$. Argumente su respuesta.

Problema 2

Sean $x$ e $y$ dos variables racionales que se relacionan mediante la igualdad $y=a^{x}$.

a) Si a es un número real cualquiera, ¿en qué caso(s) la relación es una función? Justifique en cada caso.

b) ¿Existirá un número real a para que la función del apartado a) tenga inversa? En caso afirmativo defina esta función que posee inversa.

Problema 3

Se tienen b gramos de una sustancia radiactiva la cual se desintegra a una tasa de $3 \%$ cada 10 años.

¿Cuál es la cantidad de la sustancia que queda después de t años? Muestre todo lo realizado.

Distinguimos actividad de problema porque la primera se presenta como situación rutinaria, en cambio los problemas requieren de un análisis más elaborado por parte de los informantes. Para cada una de esas instancias se presentan categorías de análisis a priori, a saber: estrategias (e), procedimientos matemáticos $(p)$ y 
argumentos (a), con un respectivo número. Se entiende por estrategia a las decisiones y acciones de carácter exploratorio que el informante crea o improvisa con el afán de probar un camino de resolución. En cuanto a los procedimientos, estos corresponden al uso de reglas, teoremas o algoritmos matemáticos de conocimiento común. Finalmente se asumen como argumentos aquellas referencias explícitas que realizan los informantes a las razones o justificaciones que los llevan a usar un procedimiento o estrategia. Las categorías a priori las establecimos considerando el conocimiento que se declaran en los programas de estudio tanto a nivel escolar como universitario para el tema función exponencial, las cuales permiten interpretar las posibles respuestas de los informantes al cuestionario, en correlato con las construcciones y mecanismos mentales que explicitamos en nuestra DG hipotética. Esa articulación de la cual hacemos mención no es algo estático, sino más bien dinámico, pues va a depender de la interpretación de los datos a la luz del método estadístico que hemos asumido.

En la Tabla 2 presentamos una descripción de cada una de esas categorías. De esta manera, cada una de ellas permite operacionalizar las componentes teóricas expresadas en la DG, facilitando el ingreso de los datos en el software CHIC versión 6.0 para obtener el árbol de similaridad y el gráfico implicativo que explicamos más adelante en el apartado metodológico.

Tabla 2. Categorías de análisis según las secciones del cuestionario.

\begin{tabular}{|l|l|}
\hline Categorías & \multicolumn{1}{c|}{ Actividad 1 } \\
\hline \multicolumn{2}{|l|}{} \\
\hline e1 & Recurre a los exponentes para expresar los productos de la multiplicación recursiva. \\
\hline e2 & Organiza y/o clasifica los cálculos de las diferencias y cocientes de las respectivas variables. \\
\hline e3 & Relaciona una diferencia con su respectivo cociente mediante un exponente. \\
\hline p1 & Utiliza la regla de la multiplicación o división de potencias de igual base. \\
\hline p2 & Escribe una igualdad para relacionar las variables de la tabla, mediante un exponente. \\
\hline p3 & Generaliza las diferencias y cocientes de las variables mediante un exponente. \\
\hline a1 & Justifica la invariante exponencial mediante propiedades de las potencias. \\
\hline \multicolumn{2}{|l}{} \\
\hline e4 & Relaciona los datos de la tabla mediante un exponente. \\
\hline p5 & Verifica que se cumple la invariante exponencial para algunos valores de la tabla. \\
\hline p6 & Hace uso de la definición del operador logaritmo para rescribir la relación de las variables. \\
\hline p7 & Aplica las propiedades del operador logaritmo. \\
\hline a2 & Hace referencia explícita a la definición del operador logaritmo \\
\hline a3 & Hace referencia explícita a las propiedades del operador logaritmo. \\
\hline \multicolumn{2}{|c}{} \\
\hline e5 & Construye un ejemplo para el caso de la raíz de índice par y la cantidad sub-radical negativa. \\
\hline e6 & Construye un ejemplo para el caso de un exponente negativo y base igual a cero. \\
\hline e7 & Esboza la gráfica de una función exponencial. \\
\hline e8 & Define una función invertible para el o los casos en que la relación sea una función. \\
\hline a4 & Hace referencia al cálculo de logaritmos \\
\hline \multicolumn{2}{|c}{} \\
\hline e9 & Analiza inductivamente el problema planteado. \\
\hline e10 & Define variables para analizar la cantidad en función del período. \\
\hline p9 & Calcula el factor para el remanente en cada período. \\
\hline a5 & Hace referencia directa a una función exponencial \\
\hline &
\end{tabular}

La interpretación de los resultados que arroja la estadística implicativa se retoma desde la teoría APOE en función de la relación entre las secciones de la DG y los grupos de elementos categoriales. Así como las categorías de análisis que se vinculan directamente con las preguntas del cuestionario, las cuales, a su vez, se relaciona con nuestra DG. A modo de ejemplo asumimos que e1, recurre a los exponentes para expresar los productos de la multiplicación recursiva, es un mecanismo que permite interiorizar la acción, multiplicar recursivamente un número 
positivo, en el proceso progresión exponente. Por otro lado p2 y e4 promueven la coordinación de los procesos, progresión exponente y variable independiente, para reconstruir la relación exponencial. Del mismo modo esperamos que e2, e3, p1, p3 y a1 ayuden a coordinar los procesos, exponente racional y relación exponente, para construir el proceso invariante exponencial. A continuación, en la Tabla 3, describimos dichas relaciones procurando establecer, de manera explícita, la relación entre los mecanismos y construcciones mentales en correlato con la actividad y los problemas del cuestionario sumando las categorías a priori que se desprenden y explicitan en la Tabla 2.

Tabla 3. Relación entre las secciones del cuestionario y las componentes de la DG.

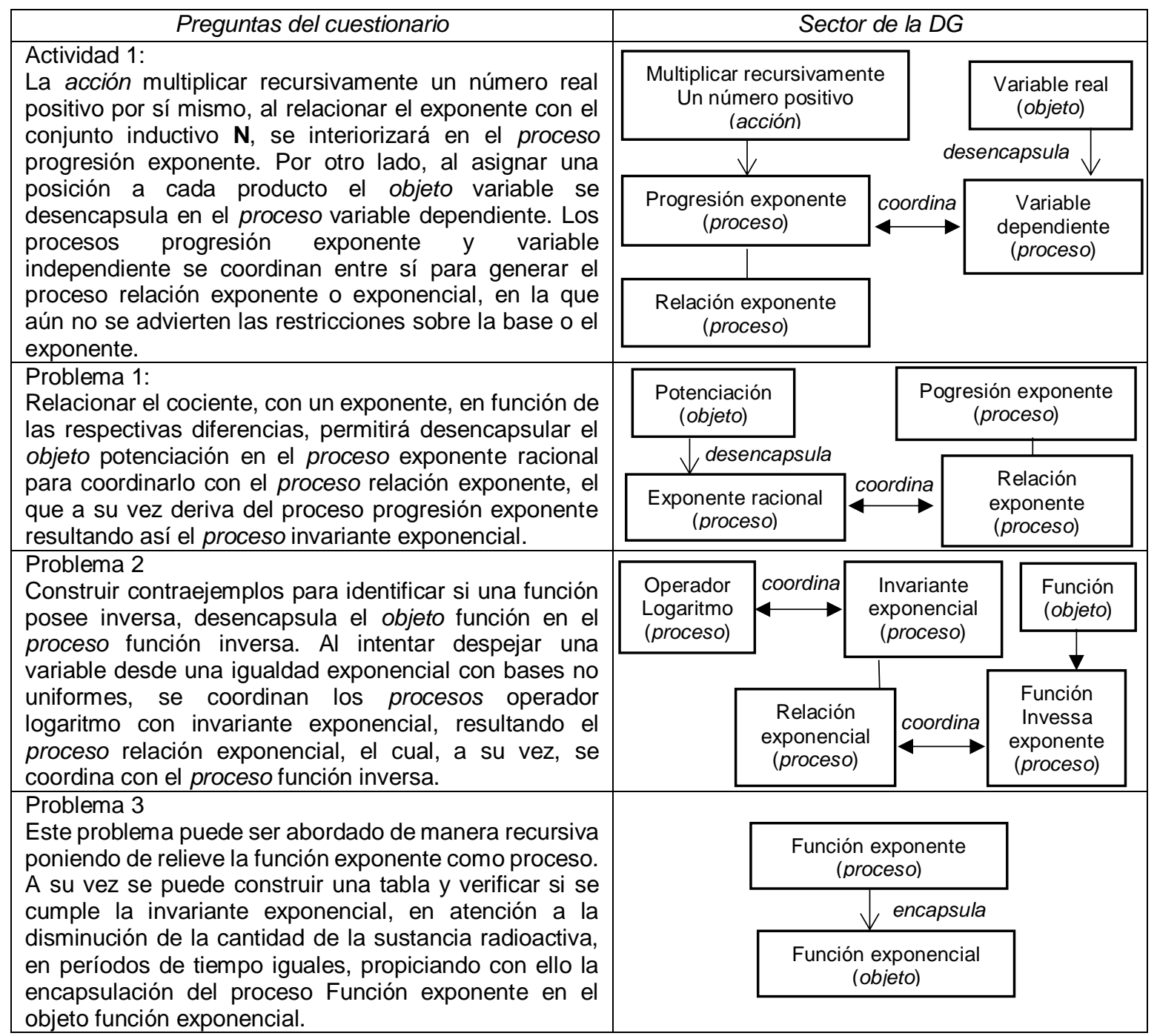

\section{Sobre el análisis de los datos}

El análisis de los datos, se sustenta en la estadística implicativa (en adelante ASI [Analyse Statistique Implicative] por su sigla en francés) (Gras et al., 2008), y el uso del software CHIC (Coherive Hierarchical Implicative Classification) versión 6.0 (Couturier, 2008). Una de las características de ASI es que combina dos tipos de análisis estadísticos multivariantes: un análisis de conglomerados (cluster analysis), para descubrir grupos homogéneos de variables, y un análisis de reglas de asociación (association rules), para explorar relaciones nosimétricas que permiten establecer relaciones de causa y efecto entre las variables analizadas. La otra característica es que ASI basa sus cálculos en la probabilidad de encontrar una coincidencia entre dos informantes (o respectivamente un contraejemplo a una regla) para medir la similitud entre las dos variables (o la intensidad de una regla que relaciona las dos variables) (Gras et al., 2008). 
El árbol jerárquico de similaridad (dendrograma) se forma de la manera habitual, esto es, comienza con cada variable formando una única clase y agrupando, a cada paso, las dos clases más similares sucesivamente, hasta llegar a una única clase que agrupa todas las variables, siendo la interpretación de los grupos hallados el aspecto más importante. Adicionalmente, el árbol de similaridad destaca algunas clases como significativas, por la relación preponderante de los índices de similaridad entre las variables que se hallan reunidas en dicha clase (Orús et al., 2009). Cuando se forma cada clase de variables similares, se puede calcular un índice de contribución de cada informante a la formación de esa clase. Con esos índices, se destaca el grupo de los informantes que más la ha manifestado. Una inspección detallada de ellos permite ahondar aún más en la descripción de la clase, así como indagar el posible efecto de otras variables (asociadas a las categorías de análisis) en la formación de dicha clase de variables similares.

El grafo implicativo es la representación gráfica de las reglas de asociación entre parejas de variables. La fuerza de las reglas de asociación se suele medir mediante un índice llamado confianza, esto es, el porcentaje de individuos que cumple la regla de entre los que la podrían cumplir (por verificar el antecedente de la regla). Este indicador es útil para predecir si un individuo presenta el rasgo del consecuente, una vez se sabe que presenta el rasgo del antecedente. No obstante, cuando el rasgo consecuente es muy abundante en los informantes, no es evidente que su presencia se deba a un efecto causado por el antecedente de la regla. Así, el grafo implicativo representa las reglas cuyas intensidades superan un umbral (elegido por los investigadores, y que suele ser del $90 \%, 95 \%$ o 99\%), y se deben interpretar como asociaciones implicativas estadísticamente significativas. A partir de estas asociaciones inesperadas, debemos distinguir relaciones de causa y efecto, que se justifican a la luz de nuestra DG hipotética, y otras relaciones, que no se podrían aprovechar si no hay una justificación adecuada.

\section{RESULTADOS}

A continuación, presentamos los resultados de aplicar el cuestionario. Para ello tabulamos las respuestas asignando un 1 a la presencia de una clase, y un 0 a la ausencia de ésta. Con la ayuda del software CHIC generamos un Árbol de Similaridad y un Grafo Implicativo para referirnos a las estructuras mentales dispuestas en nuestra DG.

\section{Sobre el árbol de similaridad en función de las categorías de análisis y la DG}

En la Figura 3 se aprecia el árbol de similaridad, que muestra la clasificación jerárquica de nuestras categorías de análisis. Hay seis clases significativas (líneas de color rojo). Las clases 1 y 6 -denotadas por (p5, a2) y ((e5 e6) p7) de arriba abajo - tienen respectivamente un índice de similaridad de 0,99 y 0,87. Para la clase 1, contribuyeron los estudiantes P2 y P8. Para la clase 6 los estudiantes P13 M3, M4, M5, M8.

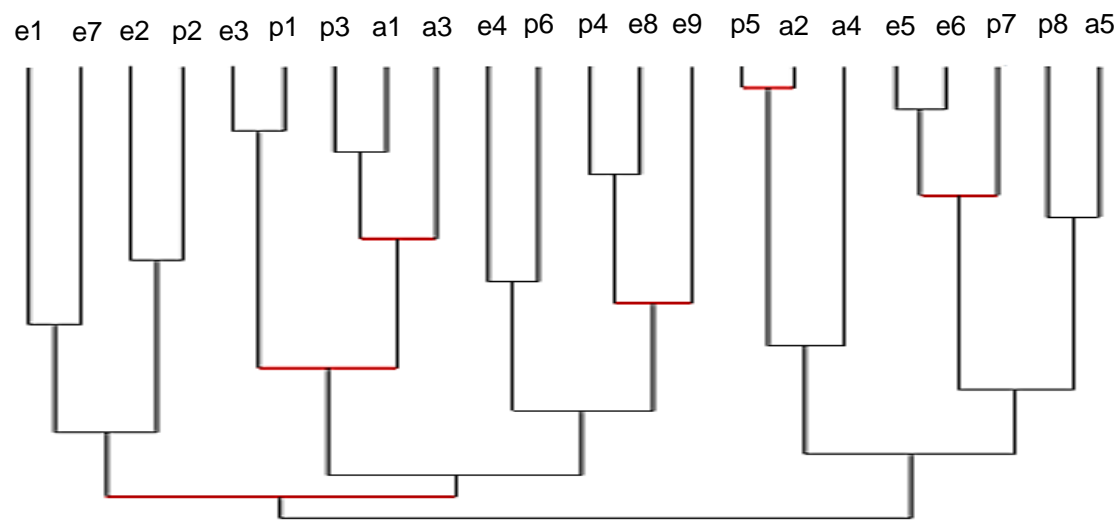

Fig. 3: Árbol de similaridad para las categorías de análisis asociadas al cuestionario

En atención a la primera clase significativa, en la Figura 4 presentamos parte de la respuesta de P2 al Problema 1 , quien relaciona las variables de la tabla que se presenta en el problema mediante exponentes, mostrando con ello una concepción acción potenciación. Además, aplica el operador logaritmo a la relación exponencial para expresar la variable dependiente en términos de la independiente, mostrando de esa manera una concepción proceso operador logaritmo. 


$$
\begin{gathered}
y=\mathrm{f}(x)=3^{x / 2}, \text { ya que } \mathrm{f}(0)=1 \text { y } \mathrm{f}(2)=1 / 3 \text { con } \mathrm{f}(-1)=3^{-1 / 2}=1 / 3^{1 / 2}=3^{1 / 2} / 3 \\
\Rightarrow \log _{3}(y)=\log 33 x / 2 \text { / por propiedad } \log _{\mathrm{a}}(a)^{x}=x \text {, además } y>0 \Rightarrow 2 \log _{3}(y)=x
\end{gathered}
$$

Fig. 4: Desempeño de P2 al Problema 1

Por otro lado, como se aprecia en la Figura 5, P8 relaciona los puntos de la tabla con la gráfica de una función exponencial y, mediante una ecuación, recurre a los datos de la tabla para determinar la base que define a dicha función. Con ello, P8 muestra una concepción proceso función exponencial. Además, utiliza la definición del operador logaritmo para expresar la variable independiente en términos de la variable dependiente, sin verificar las condiciones para realizar dicha acción, con ello muestra una concepción acción operador logaritmo.

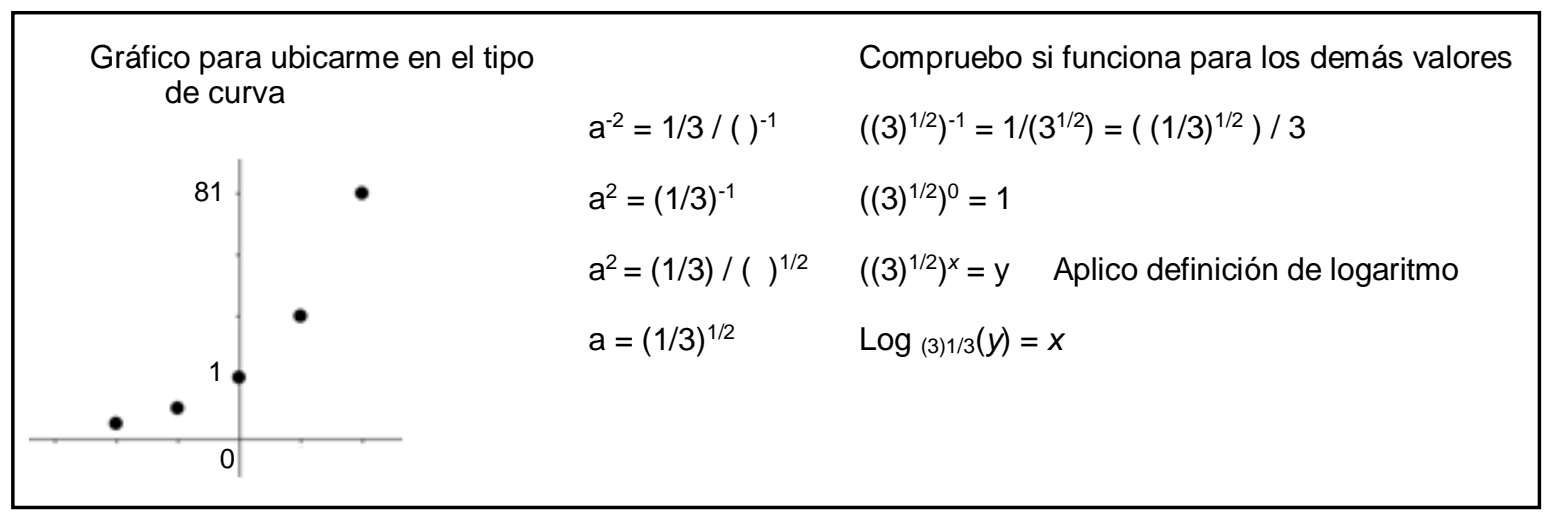

Fig. 5: Desempeño de P8 al Problema 1

En atención a la segunda clase significativa, en la Figura 6 se aprecia la respuesta de P13 al Problema 2, quien recurre a un ejemplo para establecer, de manera parcial, restricciones a la base de la relación exponencial. Pues no considera que para el valor $a=0$ y un exponente negativo no se genera un número real. Con ello, P13 muestra una concepción acción potenciación. En la respuesta de M8 al Problema 2, logra establecer los casos en que la base de la relación exponencial permite definir una función, caracterizando a cada una de ellas. Con ello, M8 muestra una concepción proceso función, como se aprecia en la Figura 7. Por último, como se aprecia en la Figura 8, M3 reescribe la igualdad mediante una propiedad del operador logaritmo y, de esa manera, establece restricciones para el parámetro a. Con ello, M3 muestra una concepción proceso operador logaritmo.

$$
\text { Con } a=-1 \text {, será } 1 \text { (par) o igual a }-1 \text { (impar). }
$$

Si $x$ es fracción tipo $1 / n, n$ par no pertenece a los reales ya que sería $y=(-1)^{1 / n}$

Con $a=0$ e $y=0$, para cualquier $x, y$ será 0 , una constante.

Fig. 6: Desempeño de P13 al Problema 2

Si $a=1 \Rightarrow y=1 \rightarrow$ Es una recta constante de pendiente cero

Si $a>1 \Rightarrow y=a^{x} \rightarrow$ Es una función exponencial de la forma:

Aproximada y es una función creciente

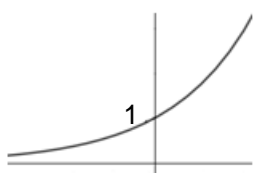

Fig. 7. Desempeño de P13 al Problema 2

Notemos que si $y=a^{x}$ se puede re-escribir como $a^{x}=e^{x \cdot \operatorname{Ln}(a)} . \operatorname{Con} \operatorname{Ln}\left(a^{x}\right)=x \cdot \operatorname{Ln}(a)$

Luego, solo tendrá sentido para $a>0$ y $x$ en los reales. Si $a=0$, no tendría sentido si $x \leq 0$.

Fig. 8: Desempeño de P13 al Problema 2 


\section{Sobre el grafo implicativo y las categorías de análisis con la DG}

En la Figura 9 se aprecia el grafo implicativo asociado a nuestras categorías de análisis. Cabe destacar que las cadenas de implicación $(\mathrm{p} 3 \rightarrow \mathrm{p} 1 \rightarrow \mathrm{e} 3)$ y $(\mathrm{a} 1 \rightarrow \mathrm{p} 1 \rightarrow \mathrm{e} 3)$ muestran, en su conjunto, aquellos procedimientos que contribuyen a la construcción proceso invariante exponencial. En general, sugieren que el cálculo de cocientes y diferencias, en la actividad del cuestionario, estimula el uso de exponentes y la división de potencias de igual base para articular la invariante exponencial con la relación exponencial.

Por otro lado, la implicación $(\mathrm{e} 6 \rightarrow \mathrm{e} 5$ ) pone de relieve dos estrategias que contribuyen a la desencapsulación del objeto potenciación en el proceso exponente racional. En particular, muestra lo pertinente de construir ejemplos que involucren exponentes racionales y negativos cuando la base es cero o bien un número real negativo. Ello contribuye directamente a la construcción de la función exponencial. A continuación, un análisis de algunas respuestas.

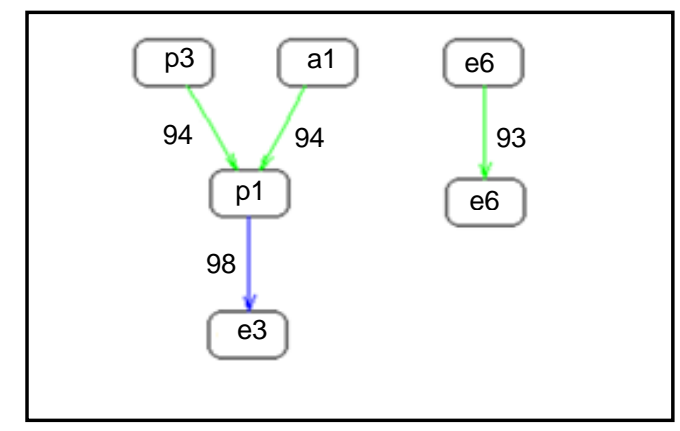

Fig. 9: Grafo implicativo de las categorías de análisis

En relación con la respuesta de P2 a la Actividad inicial del cuestionario, como se aprecia en la Figura 10, éste logra relacionar la diferencia entre dos valores de la variable independiente con el respectivo cociente de los valores de la variable dependiente, mediante un exponente. Aunque no logra generalizar dicho resultado, debido a problemas de cálculo cuando utiliza como base un número racional. Con ello, muestra una construcción acción invariante exponencial.

$$
\begin{aligned}
& \text { Caso } 2 \text { para el } 3 \text { y } 5 \quad 3-5=-2 \Leftrightarrow 343 / 15407=(7 \cdot 7 \cdot 7) /(7 \cdot 7 \cdot 7 \cdot 7 \cdot 7)=1 / 49=1 / 7^{2}=7^{-2} \\
& 5-3=\underset{\wedge}{2} \Leftrightarrow 15407 / 343=(7 \cdot 7 \cdot 7 \cdot 7 \cdot 7) /(7 \cdot 7 \cdot 7)=7^{5} / 7^{2}=7^{5-3}=7_{\uparrow}^{2}
\end{aligned}
$$

Fig. 10. Desempeño de P2 a la Actividad inicial

Por otro lado, M1 formula mediante lenguaje natural la invariante exponencial (conjetura 1) y, a su vez, relaciona potencias con raíces cuando el exponente es un número natural distinto de 1 (lo que denomina conjetura 2), como se aprecia en la Figura 11. Esa última relación, le permite abordar con éxito el Problema 1, mostrando una concepción proceso invariante exponencial y una concepción proceso potenciación. A lo anterior hay que agregar, como se muestra en la Figura 12, que M1 logra caracterizar la función exponencial, coordinando los procesos función inversa en el proceso función exponencial, mediante la visualización de la función identidad y el uso de la gráfica como mecanismos de coordinación. Sumado a ello, M1 muestra una concepción acción potenciación pues ejemplifica con determinados exponentes sin haber considerado directamente algún exponente racional.

Se considera el número 3

$\left.\begin{array}{|l|l|l|l|c|}\hline x & 1 & 2 & 3 & \begin{array}{l}3-1=2 \\ 27: 3=9\end{array}\end{array}\right\}$

Se puede afirmar que al aplicar la raíz de índice igual a la pre-imagen se obtiene la ecuación que la genera (conjetura número 2)

Fig. 11: Desempeño de M1 a la Actividad inicial 
Si $a<9$ la relación es una función $y=(-3)^{-1}=-3$

Si $a=0$ y $x \neq 0 ; y=(0)^{1}=0 ; y=0^{-1}=0$ con una constante $y=0$.

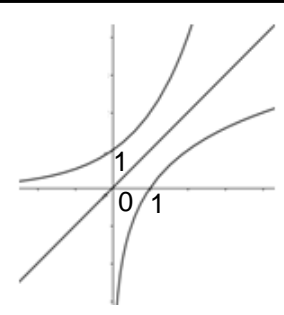

Fig. 12: Desempeño de M1 al Problema 2

\section{Algunas respuestas a los problemas 2 y 3 del cuestionario}

En la Figura 13, se presenta la respuesta de P8 al Problema 2, quien reconoce las tres posibilidades que puede asumir la base para generar una función. Llama la atención el argumento que enuncia para el caso que la base sea negativa, la alternancia de signo no genera una función y, en el caso que la base sea cero, argumenta que la gráfica se reduce a un punto. Con ello, P8 muestra una concepción acción potenciación.

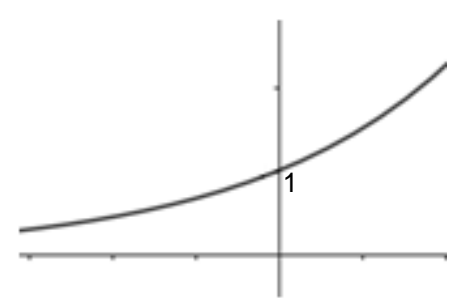

$a>1$

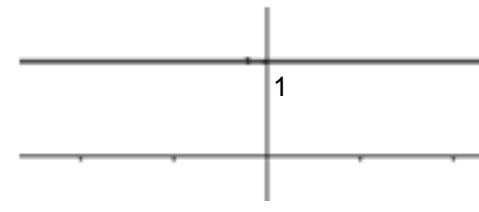

$a=1$

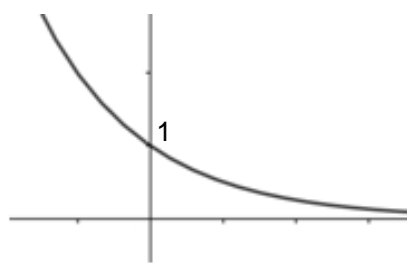

$1>a>0$

Si $a \leq 0$ no sería función ya que la relación de puntos alternos o sólo un punto ( $a=0)$.

Fig. 13: Desempeño de P8 al Problema 2

Por otro lado, en la Figura 14, P8 hace ver que la función exponencial para a > 1 posee como inversa la función logaritmo, cuyas gráficas, al parecer, son simétricas respecto de la función identidad, cuestión que no queda muy clara. Con ello, P8 muestra una concepción acción función exponencial, probablemente, dada la concepción proceso función que muestra. Para cerrar la presentación de resultados, en la Figura 15, se aprecia la respuesta de M8 al Problema 3. Como la mayoría de los informantes, hizo uso de patrones mediante exponentes, lo que muestra una concepción proceso exponenciación.

Cuando se tiene que $a>1$ se puede determinar la inversa si la función es $a^{x}=y$ su inversa será $\log _{a} x=y$

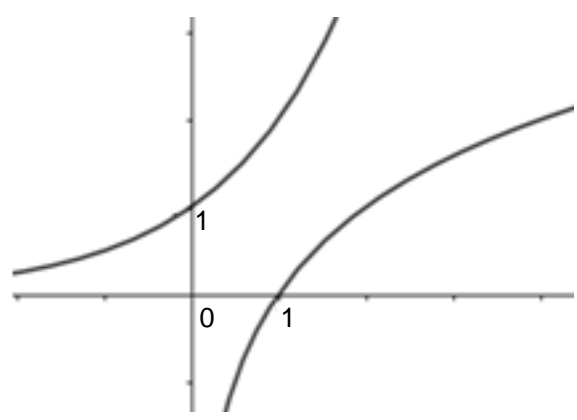

Fig. 14: Desempeño de P8 al Problema 2 


\begin{tabular}{c} 
Inicialmente: $b$ \\
+10 años: $\mathrm{b}-(3 \mathrm{~b} / 100)=b(1-3 / 100)=b \cdot(97 / 100)$ \\
+20 años: $b \cdot(97 / 100)-3(b \cdot(97 / 100)) / 100=b \cdot(97 / 100) \cdot(1-3 / 100)=b \cdot(97 / 100)^{2}$ \\
\hline
\end{tabular}

Fig. 15: Desempeño de M8 al Problema 3

Para sintetizar, presentamos en la Tabla 4 un panorama global del estado de construcción que mostró cada uno de los 22 informantes en relación a los conceptos previos y aquéllos que consideramos claves en la DG, incluyendo el de función exponencial. Esos conceptos previos o claves son: (1) variable real, (2) potenciación, (3) función, (4) invariante exponencial, (5) operador logaritmo y (6) función exponencial, en sus estados de construcción acción (A), proceso (P) u objeto (O). En la Tabla 4 se puede observar que, en el caso de los estudiantes de profesorado de secundaria, se alcanza a lo más una construcción acción de función exponencial. Sin embargo, para el caso de los estudiantes de maestría, hay una mayor adherencia a la variedad de construcciones mentales que se han propuesto en la DG.

Tabla 4. Panorama global de las construcciones mentales para el concepto función exponencial

\begin{tabular}{|c|l|l|l|l|l|l|l|l|l|l|l|l|l|}
\hline Conceptos & \multicolumn{10}{|c|}{ Estudiantes } \\
\hline & $\mathrm{P} 1$ & $\mathrm{P} 2$ & $\mathrm{P} 3$ & $\mathrm{P} 4$ & $\mathrm{P} 5$ & $\mathrm{P} 6$ & $\mathrm{P} 7$ & $\mathrm{P} 8$ & $\mathrm{P} 9$ & $\mathrm{P} 10$ & $\mathrm{P} 11$ & $\mathrm{P} 12$ & $\mathrm{P} 13$ \\
\hline 1 & $\mathrm{P}$ & $\mathrm{P}$ & $\mathrm{P}$ & $\mathrm{P}$ & $\mathrm{P}$ & $\mathrm{P}$ & $\mathrm{A}$ & $\mathrm{P}$ & $\mathrm{P}$ & $\mathrm{P}$ & $\mathrm{A}$ & $\mathrm{A}$ & $\mathrm{P}$ \\
\hline 2 & $\mathrm{~A}$ & $\mathrm{P}$ & $\mathrm{A}$ & $\mathrm{A}$ & $\mathrm{A}$ & $\mathrm{P}$ & $\mathrm{A}$ & $\mathrm{A}$ & $\mathrm{P}$ & $\mathrm{P}$ & $\mathrm{A}$ & $\mathrm{A}$ & $\mathrm{P}$ \\
\hline 3 & $\mathrm{~A}$ & $\mathrm{~A}$ & $\mathrm{~A}$ & $\mathrm{~A}$ & $\mathrm{~A}$ & $\mathrm{~A}$ & $\mathrm{~A}$ & $\mathrm{~A}$ & $\mathrm{~A}$ & $\mathrm{P}$ & - & $\mathrm{A}$ & $\mathrm{P}$ \\
\hline 4 & $\mathrm{P}$ & $\mathrm{A}$ & $\mathrm{A}$ & $\mathrm{A}$ & - & $\mathrm{P}$ & $\mathrm{A}$ & - & $\mathrm{A}$ & $\mathrm{P}$ & - & - & $\mathrm{P}$ \\
\hline 5 & $\mathrm{~A}$ & $\mathrm{~A}$ & - & - & $\mathrm{A}$ & $\mathrm{A}$ & - & $\mathrm{A}$ & $\mathrm{A}$ & $\mathrm{A}$ & $\mathrm{A}$ & $\mathrm{A}$ & $\mathrm{P}$ \\
\hline 6 & $\mathrm{~A}$ & $\mathrm{~A}$ & - & - & $\mathrm{A}$ & $\mathrm{A}$ & $\mathrm{A}$ & $\mathrm{A}$ & $\mathrm{A}$ & $\mathrm{P}$ & $\mathrm{A}$ & $\mathrm{A}$ & $\mathrm{P}$ \\
\hline & $\mathrm{M} 1$ & $\mathrm{M} 2$ & $\mathrm{M} 3$ & $\mathrm{M} 4$ & $\mathrm{M}$ & $\mathrm{M}$ & $\mathrm{M} 7$ & $\mathrm{M}$ & $\mathrm{M}$ & & & & \\
\hline 1 & $\mathrm{P}$ & $\mathrm{P}$ & $\mathrm{P}$ & $\mathrm{P}$ & $\mathrm{P}$ & $\mathrm{P}$ & $\mathrm{P}$ & $\mathrm{P}$ & $\mathrm{P}$ & & & & \\
\hline 2 & $\mathrm{P}$ & $\mathrm{P}$ & $\mathrm{A}$ & $\mathrm{O}$ & $\mathrm{O}$ & $\mathrm{P}$ & $\mathrm{P}$ & $\mathrm{P}$ & $\mathrm{P}$ & & & & \\
\hline 3 & $\mathrm{P}$ & $\mathrm{A}$ & $\mathrm{A}$ & $\mathrm{A}$ & $\mathrm{P}$ & $\mathrm{P}$ & $\mathrm{P}$ & $\mathrm{P}$ & $\mathrm{P}$ & & & & \\
\hline 4 & $\mathrm{P}$ & - & - & $\mathrm{A}$ & $\mathrm{P}$ & $\mathrm{P}$ & $\mathrm{P}$ & $\mathrm{P}$ & $\mathrm{P}$ & & & & \\
\hline 5 & $\mathrm{P}$ & $\mathrm{A}$ & $\mathrm{A}$ & $\mathrm{P}$ & $\mathrm{A}$ & $\mathrm{P}$ & $\mathrm{P}$ & $\mathrm{P}$ & $\mathrm{A}$ & & & & \\
\hline 6 & $\mathrm{P}$ & $\mathrm{A}$ & $\mathrm{A}$ & $\mathrm{P}$ & $\mathrm{P}$ & $\mathrm{P}$ & $\mathrm{P}$ & $\mathrm{P}$ & $\mathrm{P}$ & & & & \\
\hline
\end{tabular}

\section{DISCUSIÓN}

En general, hacemos notar que, desde un punto de vista metodológico, tanto la actividad como los problemas del cuestionario estuvieron acordes a los propósitos de esta investigación. Además, destacamos lo pertinente que resultó utilizar categorías de análisis, ya que con ellas fue posible vincular de forma explícita el cuestionario con los estados de construcción propuestos para la función exponencial en la DG hipotética. Sin dejar de mencionar el uso de los recursos del software CHIC, árbol de similaridad y grafo implicativo, que nos permitieron enfocar los resultados y el respectivo análisis de los datos. Respecto de nuestra DG, esta logró articular de manera coherente los aspectos centrales que proponen Weber (2002) y Vargas (2012). Esa articulación se evidenció, en parte, con algunos estudiantes, como $\mathrm{P} 13$, quien muestra evidencias de haber construido en forma paralela los procesos función exponencial y operador logaritmo. En esta dualidad de construcción, resultó relevante el proceso invariante exponencial en la construcción de la función exponencial, como lo propone Vargas (2012). De hecho, ese proceso también fue observado en las respuestas de M1 a la Actividad y el Problema 1 del cuestionario (Figura 11 y Figura 12).

Un elemento distintivo de nuestra DG es que considera como punto de partida una acción que activa el uso de exponentes y variables, elementos que permiten avanzar en la desencapsulación de los objetos potenciación y función, conceptos que, a la luz de los datos, resultan ser claves para la reconstrucción del objeto función exponencial (Figuras 4, 5, 8 y 10). Cabe resaltar que, otra característica de la DG-función exponencial, es que permite abordar el concepto de función exponencial en el ámbito del álgebra y no del análisis (en el sentido que lo plantean Montoya-Delgadillo et al., 2018), a través de una acción que contribuye, de manera auspiciosa, a una construcción proceso función exponencial, a diferencia de lo que reporta Vargas (2012). Se espera que estos elementos puedan brindar orientaciones a la práctica del docente universitario, en la medida que argumentan la importancia de retomar el concepto función exponencial a partir de acciones cognitivas simples, que le permitan a los estudiantes reconstruir conocimientos previos, combinando el ámbito aritmético con el algebraico. 
En relación con la primera pregunta de investigación, destacan las estrategias asociadas a la multiplicación recursiva, pues el proceso invariante exponencial se gesta a partir de la interiorización de la acción multiplicar recursivamente un número real positivo, mediante el uso de exponentes enteros positivos como mecanismo de interiorización. Lo anterior se refleja en la respuesta de M1 y a la Actividad del cuestionario (Figura 11), quien relaciona, mediante una igualdad, un exponente con las dos variables de la tabla. Luego, en el Problema 1, logra hallar una solución utilizando una de las dos conjeturas que formula.

Por otro lado, para reconstruir el objeto función exponencial es necesario contar con el objeto potenciación pues, para determinar aquellas condiciones de la base y el exponente de la relación exponencial, se necesita definir aquellos casos, mediante ejemplos que muestren la imposibilidad de obtener alguna imagen del dominio de la variable, dificultades que se manifestaron en M3 y P13 (Figura 8 y Figura 9), pese a tener un buen desempeño en el cuestionario. Además, es clave el objeto función, ya que permite determinar aquellos casos que definen dos funciones que poseen inversa, aspecto que se muestra con claridad en la respuesta de M1. Aunque M1 no logra manifestar un estado de construcción mental objeto de la función exponencial, dado que sólo muestra una construcción acción invariante exponencial, sí evidencia una comprensión gráfica de la inversión de la función exponencial. Prueba de lo anterior es la forma como M1 aborda los Problemas 1 y 3.

Para nuestra segunda pregunta de investigación, sobresale el hecho que muchos de los estudiantes, no habiendo mostrado el proceso invariante exponencial, logran obtener, mediante el uso de patrones y las propiedades de las potencias, una expresión analítica de las variables asociadas a una tabla que tiene relación con la función exponencial. La fundamentación de ese procedimiento matemático se basa en que la función exponencial es un isomorfismo de un grupo aditivo $\mathrm{R}$ hacia uno multiplicativo $\mathrm{R}^{+}$, que cumple con la propiedad $f(a+b)=f(a) \cdot f(b)$ (Guerrero-Ortiz et al., 2018), y su utilización también fue considerada en la propuesta de Vargas (2012), pero desde la interiorización de acciones. Esto último es relevante porque dicha propiedad es exclusiva de la función exponencial y de su inversa, la función logarítmica, la que caracteriza aspectos intrínsecos de su comportamiento como función continua y derivable (Kuzniak et al., 2017). Todo ello pone de relieve el valor que adquieren los procedimientos más elementales en la formación de los estudiantes del profesorado para cimentar esa matemática universitaria de manera más significativa. En relación con las clases significativas de nuestro árbol de similaridad, destaca la primera clase que explicita un razonamiento recursivo para vincular diferencias con cocientes mediante un exponente.

La segunda clase, esencialmente apunta a la concatenación de estrategias, procedimientos y argumentos que describen, respectivamente, la construcción de la exponencial como una invariante, quedando fuera la aplicación de la exponencial como un modelo de comportamiento, en el que por cada unidad de tiempo se tiene un decrecimiento en una razón constante (Figura 15). Estos resultados reafirman la importancia de la construcción covariacional de la exponencial, que promueve inicialmente la coordinación entre aspectos aditivos del conteo con aspectos multiplicativos de sucesiones racionales (Ferrari-Escolá et al., 2016). Por último, cabe mencionar el rol que juegan la elaboración de una tabla de valores en la construcción del proceso invariante exponencial, así como la elaboración de una gráfica en la construcción objeto función exponencial, estos recursos pueden considerarse, en el sentido de da Silva y de Almeida (2018), signos que producen los estudiantes para atribuir significados específicos a la función exponencial según el tipo de actividad que se resuelve. Estos elementos que no fueron considerados en nuestra DG, lo que se asume como un hallazgo a tener en cuenta en la refinación de ella.

\section{CONCLUSIONES}

Los resultados del estudio permiten validar el modelo cognitivo propuesto en la DG, vindicando los elementos teóricos que la sustentan. A la luz de la discusión que se ha planteado, cabe destacar el rol que tiene organizar la multiplicación recursiva de un número real positivo en una lista o tabla de valores, como una estrategia didáctica para aproximarse de manera discreta a la función exponencial desde un punto de vista no-mecanicista. Dicha estrategia didáctica permitió activar diferentes estrategias y procedimientos matemáticos que ayudaron a articular la matemática en torno a la función exponencial. Por otro lado, con este estudio se pone de relieve lo necesario de promover procesos inductivos desde secuencias de tareas que permitan la aplicación de procedimientos matemáticos seleccionados auténticamente por parte de un aprendiz y, a la vez, la utilización libre de estrategias, para evitar con ello respuestas prototípicas aprendidas de memoria, que son evidencia de una comprensión superficial de un concepto o noción matemática por parte de un estudiante. Por último, un aspecto que emerge en este estudio, y que aporta a la refinación de la DG, es que el proceso operador logaritmo se construye a la par con el proceso relación exponencial mediante la propiedad invariante de la exponencial, facilitando con ello que la función exponencial y la función logaritmo surjan paralelamente. 


\section{AGRADECIMIENTOS}

Los autores agradecen el apoyo del proyecto Fondecyt №11190152 y la colaboración de las Facultades de Educación de la Universidad de Playa Ancha y Universidad Academia Humanismo Cristiano. Además del Centro de Estudios Avanzados de la UPLA y el Instituto de Matemática de la PUCV.

\section{REFERENCIAS}

Arnon, I., Cottrill, J., y otros cinco autores, APOS theory: a framework for research and curriculum development in Mathematics Education, Springer, Nueva York, United States (2014)

Bryan, J., Using radioactive decay to investigate exponential functions, https://www.jstor.org/, The Mathematics Teacher, 106 (1), 52-58 (2012)

Bush, S., Gibbons, K., Karp, K., y Dillon, F., Epidemics, exponential functions, and modelling, https://www.jstor.org/, Mathematics Teaching in the Middle School, 21(2), 90-97 (2015)

Cajori, F., History of the exponential and logarithmic concepts, https://doi.org/10.1080/00029890.1913.11997915, The American Mathematical Monthly, 20(2), 35-47 (1913)

Confrey, J., The concept of exponential functions: a student's perspective, en L. Steffe (Ed.), Epistemological foundations of mathematical experience, 124-159, Springer-Verlag, Athens, United States (1991)

Confrey, J., y Smith, E., Exponential functions, rates of change, and the multiplicative unit, https://doi.org/10.1007/bf01273661, Educational Studies in Mathematics, 26(2-3), 135-164 (1994)

Cordero, F., y Miranda, E., El entendimiento de la transformada de Laplace: una epistemología como base de una descomposición genética, RELIME: Revista Latinoamericana de Investigación en Matemática Educativa, 5(2), 133-168 (2002)

Couturier, R., CHIC: cohesive hierarchical implicative classification, en R. Gras, E. Suzuki, F. Guillet y F. Spagnolo (Eds.), Statistical implicative analysis: theory and applications, 41-53, Springer, Berlín-Heidelberg, Germany (2008)

da Silva, K., y de Almeida, L., The exponential function meaning on mathematical modeling activities: a semiotic approach, https://doi.org/10.17583/redimat.2018.2762, Journal of Research in Mathematics Education, 7(2), 195-215 (2018)

Dennis, D., y Confrey, J., The creation of continuous exponents: a study of the methods and epistemology of John Wallis, https://doi.org/10.1090/cbmath/006/02, Research in Collegiate Mathematics, II, 33-60 (1996)

Dubinsky, E., Reflexive abstraction in advanced mathematical thinking, en D. Tall (Ed.), Advanced mathematical thinking, 95-123, Springer, Nueva York, United States (1991)

Dubinsky, E., y Harel, G., The nature of the process conception of function, en G. Harel y E. Dubinsky (Eds.), The concept of function: Aspects of epistemology and pedagogy, MAA Notes 25, 85-106, Mathematical Association of America Washington, Washington, United States (1992)

Dubinsky, E., y Lewin, P., Reflective abstraction and mathematics education: the genetic decomposition of induction and compactness, Journal of Mathematical Behavior, 5(1), 55-92 (1986)

Ellis, A. B., Özgür, Z., y otros tres autores, Quantifying exponential growth: three conceptual shifts in coordinating multiplicative and additive growth, https://doi.org/10.1016/j.jmathb.2015.06.004, The Journal of Mathematical Behavior, 39, 135-155 (2015)

Eves, H., An introduction to the history of mathematics, fourth edition, Holt. Rinehart, and Winston, New York, United States (1976)

Ferrari, M., y Farfán, R. M., Una socioepistemología de lo logarítmico, RELIME: Revista Latinoamericana de Investigación en Matemática Educativa, 13(4-I), 56-68 (2010)

Ferrari-Escolá, M., Martínez-Sierra, G., y Méndez-Guevara, M. E. M., Multiply by adding: development of logarithmicexponential covariational reasoning in high school students, https://doi.org/10.1016/j.jmathb.2016.03.003, The Journal of Mathematical Behavior, 42, 92-108 (2016)

González, D., y Roa-Fuentes, S., Un esquema de transformación lineal: construcción de objetos abstractos a partir de la interiorización de acciones concretas, https://doi.org/10.5565/rev/ensciencias.2150, Enseñanza de las Ciencias, 35(2), 89107 (2017)

Gras, R., Suzuki, E., Guillet, F., y Spagnolo, F., Statistical implicative analysis: theory and applications, Springer, BerlínHeidelberg, Germany (2008) 
Guerrero-Ortiz, C., Mena-Lorca, J., y Morales-Soto, A., Fostering transit between real world and mathematical world: some phases on the modelling cycle, https://doi.org/10.1007/s10763-017-9856-9, International Journal of Science and Mathematics Education, 16(8), 1605-1628 (2018)

Kuzniak, A., Tanguay, D. y otros cuatro autores, Conectar los ETM del análisis: el caso de la función exponencial, en I. M. Gómez-Chacón, A. Kuzniak, K. Nikolantonakis, P. R. Richard y L. Vivier (Eds.), Proceedings Fifth ETM Symposium, 49-62, University of Western Macedonia, Florina, Greece (2017)

Martínez-Sierra, G.M., Explicación sistémica de fenómenos didácticos ligados a las convenciones matemáticas de los exponentes. Revista Latinoamericana de Investigación en Matemática Educativa, RELIME: Revista Latinoamericana de Investigación en Matemática Educativa, 5(1), 45-78 (2002)

Montoya-Delgadillo, E., Páez-Murillo, R., Vandebrouck, F., y Vivier, L., Deconstruction with localization perspective in the learning of analysis, https://doi.org/10.1007/s40753-017-0068-z, International Journal of Research in Undergraduate Mathematics Education, 4(1), 139-160 (2018)

Orús, P., Zamora, L., y Gregori, P., Teoría y aplicaciones del análisis estadístico implicativo: primera aproximación en lengua hispana, Castellón, España: Departamento de Matemáticas Universidad Jaume I, Santiago, Cuba: Facultad de Matemática y Computación Universidad de Oriente de Santiago de Cuba (2009)

Presmeg, N., y Nenduradu, R., An investigation of a preservice teacher's use of representations in solving algebraic problems involving exponential relationships, en H. L. Chick y J. L. Vicent (Eds.), Proceedings of the 29th Conference of the International Group for the Psychology of Mathematics Education, 4, 105-112, PME, Melbourne, Australia (2005)

Rodríguez, G., Gil, J., y García, E., Metodología de la Investigación cualitativa (2da ed.), Aljibe, Málaga, España (1999)

Rodríguez, M. A., Mena A., y otros tres autores, Construcción cognitiva del conjunto solución de un sistema de ecuaciones lineales con dos incógnitas, https://doi.org/10.5565/rev/ensciencias.2194, Enseñanza de las Ciencias, Revista de Investigación y Experiencias Didácticas, 37(1), 71-92 (2019)

Stake, R. E., Qualitative research: studying how things work, The Guilford Press, Nueva York, United States (2010)

Vargas, J., Análisis de la práctica del docente universitario de precálculo, Estudio de casos en la enseñanza de las funciones exponenciales, https://gredos.usal.es/handle/10366/121430, Tesis doctoral, Universidad de Salamanca, España

Weber, K., Students' understanding of exponential and logarithmic functions, Proceedings of the 2nd International Conference on the Teaching of Mathematics (at the undergraduate level), 1-7, John Wiley y Sons, Hersonissos, Greece 